\title{
Dynamical evolution of extrasolar planetary systems
}

\author{
Ji-Lin Zhou and Yi-Sui Sun \\ Department of Astronomy, Nanjing University, Nanjing 210093, China \\ email: zhoujl@nju.edu.cn,sunys@nju.edu.cn.
}

\begin{abstract}
To date, more than 130 extrasolar planets around main sequence stars are revealed mainly by the Doppler radial velocity measurements. Due to the observational biases, most of the detected planets are moving in orbits close to the host stars, with some in highly eccentric orbits. Dynamical processes during the late stage of planet formation are important to account for the present orbital properties. These processes include: planet migrations and resonance trappings caused by gravitational interactions between protostellar disk and planets, dynamical scattering due to interactions between planets, etc. In this paper, we review the major effects of these dynamical processes on the orbital characteristics of the planet systems.
\end{abstract}

Keywords. Celestial mechanics, stars: planetary systems

\section{Introduction}

The study of dynamical evolution of planets under mutual gravitational interactions is a classic subject for celestial mechanics, which can be backdated to the works of Laplace and Lagrange in the late eighteen's century. However, as the solar system was the only paradigm of planetary system before 1990's, the classical perturbation theory for planet dynamics was based on near-circular orbits with large separations. The discovery of the extrasolar planets around a neutron star PSR $1257+12$ by Wolszczan \& Frail (1992) and a solar-type star 51 Peg by Mayor \& Queloz (1995) opened a new era of planetary science. To date, more than 130 extrasolar planets around solar-type stars have been discovered $(\dagger)$, among them 11 multiple planetary systems are confirmed. As most of the discovered extrasolar planets are moving in orbits close to the host stars, with some in high eccentricity orbits, both the classical planet formation theories and the classical perturbation and stability theories are facing great challenges.

There have been many excellent reviews on the dynamical evolution of extrasolar planet systems during the past years, e.g., Lin et al. (2000), Ward \& Hahn (2000), et al.. In this paper, we collect some of the current knowledge on the this subject. First we make some statistics on the orbital distributions of the discovered extrasolar planets, then we review some important dynamical processes as well as some recent progresses that are helpful to understand these characteristics.

\section{Statistics of Extrasolar Planets}

We use the elements of 114 planets, where 111 planets are taken from the web (exoplants.org), and the rest 3 are from the recently discovered "very hot Jupiters": OGLE-TR-56b (Torres et al. 2004), OGLE-TR-113b (Konacki et al. 2004), OGLE-TR132b (Bouchy et al. 2004). The orbital distributions of these planets show the following statistical properties (see also Udry, Mayor \& Queloz 2003, Marcy et al. 2003):

$\dagger$ See http://www.obspm.fr/encycl/encycl.html, http://exoplanets.org/ 

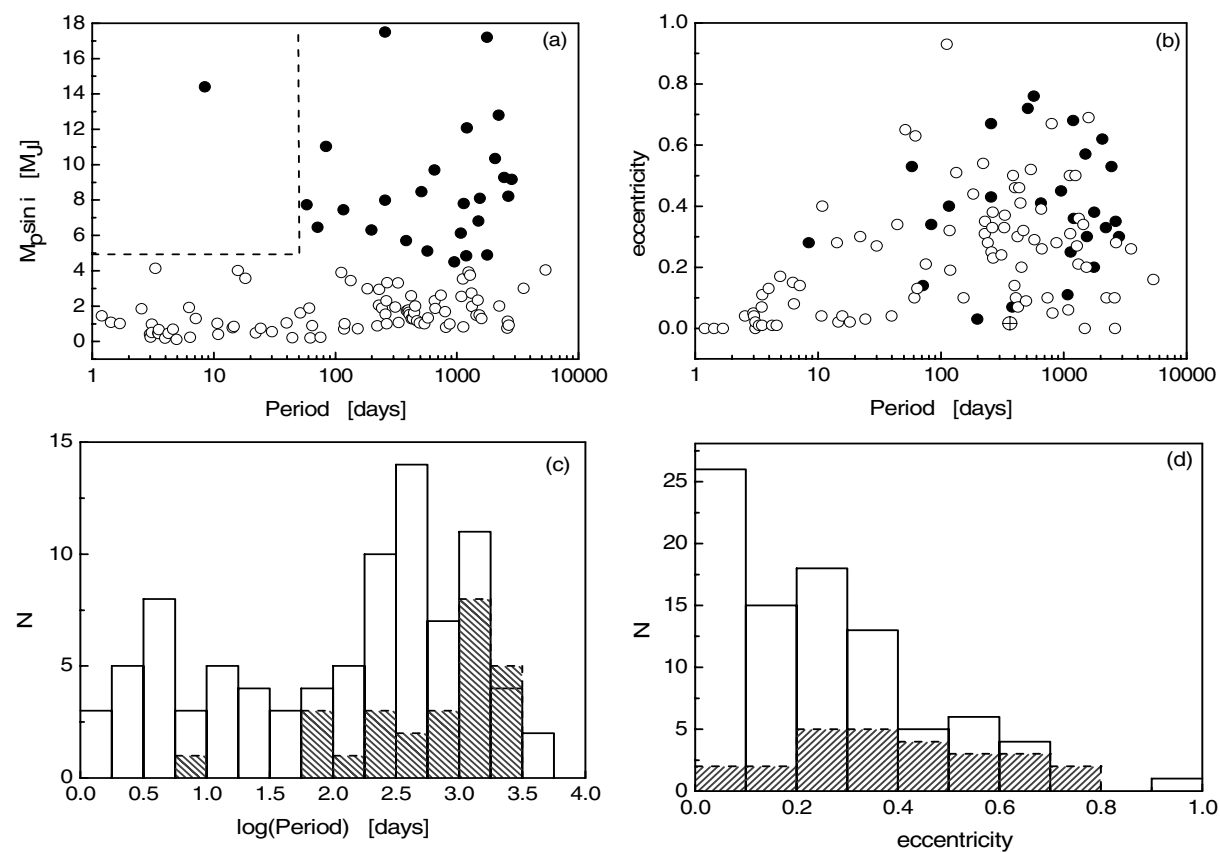

Figure 1. Statistical graphes for the dynamical parameters of the observed 114 planets (see text). In (a) and (b): open circles are for planets with $M_{p} \sin i<5 M_{J}$ (total No.=88), and filled circles for those with $M_{p} \sin i>5 M_{J}$ (total No.=26). The dotted horizontal (vertical) line in (a) corresponds to $M_{p} \sin i=5 M_{J}$ (period=50days, resp.). In (c) and (d): the distributions with empty (shaded) bars are for planets with $M_{p} \sin i<5 M_{J}\left(M_{p} \sin i>5 M_{J}\right.$, resp.).

Period-mass graph. Due to the limitation of the observations, most of the planets are located in short period orbits with masses comparable to Jupiter(Fig.1a). If we classify the planets into two groups: large planets $\left(M_{p} \sin i>5 M_{J}\right)$ and small planets $\left(M_{p} \sin i \leqslant 5 M_{J}\right)$, then almost all large planets are located in orbits with $P>50$ days $(\sim 0.27 \mathrm{AU})$ (Fig.2b). The only one exception at present time is the planet of HD162020, with $M_{p} \sin i=14.4 M_{J}$, and a period of 8.4 days. This phenomenon may be the consequence of either the inefficient accretion of planets at small radius during the late stage of formation, or the dependence of migration speed on planet masses after their formation. We will discuss this later.

Period-eccentricity graph. Both larger planets and small planets can reach high eccentricities, indicating that there are some robust mechanisms to pump planet eccentricities (Fig.1b). The tidal circularization time scale is $\sim 5.4 \mathrm{Gyr}$ for a Jupiter mass planet around a solar mass star in a 3-day period orbit (Lin et al. 2000), thus the eccentricities of planets in orbits with periods $<3$ days were most possibly damped. The detection of the three "very hot Jupiters" reduces the "cutoff" period to 1day $(\sim 0.02 \mathrm{AU})$.

Period distribution. There is a slight shortage of small planets at intermediate period $(\sim 10-200$ days) orbits (Fig.1c). Large planets locate somewhat evenly in orbits with period $100 \sim 3000$ days, except a small peak at period $\sim 1000$ days.

Eccentricity distribution. The eccentricity distribution of the 26 large planets has an average at 0.39 , with a variance 0.2 (Fig.1d). For the 88 small planets, the average value is 0.23 , with a variance 0.19 . The large difference of the average eccentricities between large and small planets shows that they might have passed some different dynamical histories. 


\section{Dynamics of Single Planet Systems}

We begin with a planet system which is in the late stage of planet formation, so at least one protoplanet (or planet core) was formed in a gaseous disk, together with a planetesimals disk in the equatorial plane of the host star. It is generally believed that short-period planets were formed several AU away from their host stars and subsequently migrated to their present locations (Lin, Bodenheimer \& Richardson 1996). On the other hand, orbital migration is a natural consequence of the interactions between gaseous disk and planets.

When the protoplanets formed through core accretion scenario, dynamical frictions with the residual populations of smaller bodies reduce the orbital eccentricities and inclinations of the protoplanets, which results nearly circular, coplanar orbits for the protoplanets (Kokobu \& Ida, 1998). Thus the eccentricities of the observed planets could, most probably, be excited after the protoplanets were formed.

\section{1. planet-Gaseous disk interactions}

A protoplanet perturbs its nascent gaseous disk through gravitation. According to the linear theory, the protoplanet exerts torques on a dynamically cold disk $(c \ll r \Omega$, where $c, r, \Omega$ are the sound speed of gas, the orbital radius and angular velocity of planet motion, respectively) mainly at the Lindblad and corotation resonances (Goldreich and Tremaine, $1979,1980)$. In a Keplerian disk(and hereafter), these resonances occur at locations with $\Omega(r): \Omega_{p}=l:(m+\epsilon)$, where $\Omega_{p}$ is the angular velocity of the protoplanet, $l, m$ are integers, $|l-m|$ is the order of the resonance, $\epsilon=-1,0,1$ correspond to the inner Lindblad resonances (ILRs), corotation resonances(CRs), and outer Lindblad resonances (OLRs), respectively. The torque of a protoplanet exerts on the disk at a Lindblad resonance (LR) is given as (Goldreich and Tremaine, 1980),

$$
T_{l, m}^{L} \approx \frac{\pi^{2}}{3 \epsilon}\left[\sigma \Omega^{-2}\left(\frac{r d \psi_{l, m}}{d r}-\frac{2 m}{\epsilon} \psi_{l, m}\right)^{2}\right]_{r=r_{L}}
$$

and that at a corotation resonance $(\mathrm{CR})$ is given as,

$$
T_{l, m}^{C}=-\frac{4}{3} m \pi^{2}\left[\left(\frac{r}{\Omega}\right) \psi_{l, m}^{2} \frac{d}{d r}\left(\frac{\sigma}{\Omega}\right)\right]_{r=r_{C}}
$$

where $\sigma$ is the surface density of the disk, $\psi_{l, m} \sim O\left(\mu e^{|l-m|}\right)$ is the coefficient of the protoplanet potential under Fourier expansion, and $\mu=M_{p} / M_{*}$ where $M_{*}$ is the mass of the star. Thus the torques at LRs have the same sign of $\epsilon$, while those at CRs depend on the gradient of $\sigma / \Omega$.

\subsubsection{Orbital migrations}

There are two main types of migrations for a protoplanet under tidal interaction with the gaseous disk:

Type I migration. Suppose the protoplanet is too small to make large modifications to the surface density of the disk, and the disk has surface density like the minimummass solar nebula, where $\sigma \sim r^{-3 / 2} \sim \Omega$. in such disks the torques at CRs (3.2) vanish in an almost unperturbed disk (Ward 1988, Artymowicz 1993). The differential torques generated by LRs between the inner and outer part (relative to the protoplanet orbit) of the disk drive the protoplanet to migrate inward, which is called type I migration (Ward 1997a). The time scale for the orbital decay, $\tau_{I}=r / \dot{r}$, is given by (Ward 1997b)

$$
\tau_{I} \sim \frac{1}{2 \pi c_{1}}\left(\frac{M_{*}}{M_{p}}\right)\left(\frac{M_{*}}{\sigma r^{2}}\right)\left(\frac{c}{r \Omega_{p}}\right)^{3} T_{p}
$$


Where $c_{1}$ is a constant of order unit, $T_{p}=2 \pi / \Omega_{p}$ is the orbital period of the planet. Setting $M_{*}=M_{\odot}, c / \Omega_{p} \sim h=0.07 r$, where $h$ the scale height of the disk, $\sigma r^{2}=$ $10^{-3} M_{\odot}$, thus for a planet with mass $10 M_{\oplus}$ at $r=5 \mathrm{AU}$, one has $\tau_{I} \approx 2 \times 10^{4}$ years.

Gap formation. As the torques at ILRs (OLRs) are negative (positive,respectively), the inner disk loses angular momentum while the outer disk gains it. Hence, a protoplanet tends to form a gap near it, while disk viscosity tends to close it. The critical mass of the protoplanet that can open a gap is (Lin and Papaloizou 1979, Lin and Papaloizou 1993),

$$
\mu_{c}=\frac{40 \nu}{r_{p}^{2} \Omega_{p}}=40 \alpha\left(\frac{c}{r_{p} \Omega_{p}}\right)^{2}
$$

where we have used the $\alpha$ as prescribed by the kinematic viscosity (Shakura-Sunyaev 1973), $\nu \sim \alpha c^{2} / \Omega$. With the above parameters, and set $\alpha \sim 10^{-4}$, we have $m_{\text {gap }}=$ $\mu_{c} M_{\odot} \approx 7 M_{\oplus}$. Hydrodynamical simulations show that the above criterion is accurate within a factor of 2 (Bryden et al. 1999). It was believed that gap formation will reduce greatly the mass accretion of the protoplanet (Lin and Papaloizou 1993). Hydrodynamical simulation found the mass accretion rate through the gap onto the planet is reduced markedly when the disk viscosity is $\operatorname{small}\left(\alpha \leqslant 5 \times 10^{-4}\right)$, but when the disk has large viscosity $\left(\alpha \sim 10^{-3}\right)$, the protoplanet is still able to accrete lots of mass even after the formation of a nearby gap (Kley 1999).

Type II migration. When a gap is maintained near a protoplanet, the tidal torques at resonances balance the viscosity torque. Thus the protoplanet is locked to the disk, and will drift inward with a time scale determined by the viscosity of the disk:

$$
\tau_{\nu}=\frac{r^{2}}{\nu} \sim \frac{1}{2 \pi \alpha}\left(\frac{r \Omega_{p}}{c}\right)^{2} T_{p}
$$

Adopting $\alpha=10^{-4}$ and $c /(r \Omega)=0.07$, we find a planet at $5 \mathrm{AU}$ will drift inward with a time scale of $\tau_{\nu} \approx 3 \times 10^{6}$ years.

\subsubsection{Problems on planet migration}

Time scale of migration. Both type I and type II migrations predict fast inward migrations for a protoplanet after it is formed, especially the type I migration has a time scale of $\sim 10^{4}$ years, which is much shorter than the age of the gaseous disk $\left(\sim 10^{7}\right.$ years $)$. To reduce the risk of migrating planets swallowed by the host star, a possible solution is trying to reduce the minimum mass for gap formation, thus reduce the time of a planet in type I drift. Considering the nonlinear evolution of the density waves in a thick disk could slightly reduce $m_{\text {gap }}$ to $2-15 M_{\oplus}$ (Rafikov 2002). Koller, Li \& Lin (2003) propose that secondary shearing instabilities will occur near the streamline separatrix at $3.5-5$ Hill's radius away form the planet, which can lead to the formation of large vortices and thus results in fluctuating (positive and negative) torques instead of the negative ones. Laughlin, Steinacker and Adams (2004), Nelson and Papaloizou (2004) argue that, in the locations where the magnetorotational instability is active, type I migration can be readily overwhelmed by turbulent perturbations, and gravitational torques arising from magnetohydrodynamics turbulence will contribute a random walk component to the migratory evolution of the planets, thus increasing the drift time scale.

The inward migration of a planet could be stopped near the star as a result of tidal interaction with the host star, or through the evacuation of the inner disk by some processes such as interactions with stellar magnetosphere (Lin, Bodenheimer \& Richardson 1996), magnetorotational instability (Kuchner \& Lecar 2002), photoevaporation by irradiation from the central star (Matsuyama, Johnstone and Murray 2003), etc. The negligible 
eccentricity of all extra-solar planets with periods less than 6 days can be accounted for by tidal dissipation induced by their host stars. While the coexistence of planets with periods 7-21 days on both circular and eccentricity can be accounted for by the variations in spin-down rates of the young stars (Dobbs-Dixon, Lin, Mardling, 2004)

Lack of large planets on small period orbits. The observed lack of large planets on small period orbits, if it is a true phenomenon, needs an explanation (Fig.1a). Tidal interactions between host stars and the protoplanets cannot account for this, since the orbital decay timescale would be $\sim 10^{12}$ years for a Jupiter mass planet in an orbit with period of 10 days (Terquem et al. 1998, Lin et al. 2000). Type II migration alone can not account for this either, since the migration speed is independent of the protoplanet mass. Ivanov, Papaloizou \& Polnarev (1999) found that, when the mass of the planet is larger than the characteristic mass with which it tidally interacts, the inertia of the planet becomes important in slowing down the orbital evolution. This model predicts that a protoplanet with mass substantially larger than $M_{d 0}$, the mass of disk inside the planet orbit, should not increase its mass significantly before migrating to the center of the disc. Thus the planet mass should be limited at about $M_{d 0} \sim \sigma r^{2}$, which implies that planets with comparatively large period orbits could have larger masses. Numerical results of Nelson et al. (2000) show that the maximum mass of a protoplanet that can accretion while migrating to the neighborhood of the central star would be $\sim 5 M_{J}$.

Direction of planet migrations. While most of the theories and numerical results predict inward migrations, an outward scenario is required by the existence of planets with large semi-major axis (like Uranus and Neptune). This because, according to the core formation scenario, the time scale for the planets to form at larger semi-major axis (several tens of AU) would be prohibitively long. Outward migrations can be caused also by interactions with gas disk in some circumstances. Veras \& Armitage (2004) proposed that, a strong mass loss from the disk can cause a planet to migrate outward. The strong mass loss from the disk can be caused by photoevaporation from the host star if it is more massive than the Sun, or result from removal by other perturbations from nearby stars within a cluster. Other mechanisms such as interactions between planet and planetesimal disc (see Sec.3.2) and protoplanets scattering can also cause outward migrations.

\subsubsection{Eccentricity evolution}

The linear theory predicts that LRs can excite the eccentricity of a protoplanet in the disk, while CRs damp the eccentricity. Thus the final evolution of the eccentricity depends on the competitions between these two effects. However, as the magnitudes of the CRs depend on the gradient of the local surface density of the disk, the final evolution of planet eccentricity may be quite elusive (Goldreich \& Tremaine 1980, Lin and Papaloizou 1993). Briefly speaking, it depends on the circumstances of the disk and planet:

Small planet case. If a protoplanet is too small to open a gap near it $\left(\mu \sim 10^{-6}\right)$, the CRs are not important. The role of the LRs on the protoplanet depends on their locations. According to Ward (1988), LRs outside (both interior and exterior) it's orbit excite planet eccentricity, while those co-orbiting with the protoplanet damp the eccentricity more effectively. Thus the eccentricity of the protoplanet ultimately suffers decay.

Moderate planet case. If the protoplanet is large enough to open a gap near it $(\mu=$ $10^{-5} \sim 10^{-4}$ ), the gradient of $\sigma / \Omega$ is large near the gap, so the CRs dominate by a slight margin, and the eccentricity is damped (Goldreich \& Tremaine 1980).

Massive planet case. When the protoplanet is massive $\left(\mu \sim 10^{-3}\right)$, the gap near it is wide enough so that all the important resonances (with large $m$ ) fall into the gap, thus both LRs and CRs can be severely weakened, in this case the increase of eccentricity is possible. Lin and Papaloizou (1993) show both analytically and numerically that, for 
a massive planet with its gap truncated near the $2: 1$ resonance, it's eccentricity may increase with a rate,

$$
\tau \sim\left(\frac{1}{\mu} \frac{M_{*}}{\pi \sigma r_{p}^{2}}\right) T_{p}
$$

So for a Jupiter mass planet at 5AU, suppose $\pi \sigma r_{p}^{2} \sim 10^{-3} M_{*}$, its eccentricity could increase in $10^{7}$ years, the same time scale as the age of the gaseous disk. Goldreich \& Sari (2003) shows the evolution of the disk and a massive planet could lead to a increase of planet eccentricity. Moreover, the depletion of the disk can also cause changes of planet eccentricities (Nagasawa, Lin \& Ida 2003).

Numerical simulations give similar qualitative results, i.e., the eccentricity of planets with large masses will increase under planet-disk interactions, but those with small masses may have their eccentricity damped. With two-dimensional hydrodynamical simulations, Papaloizou, Nelson and Masset (2001) shows that, there exist a transition mass $\left(\sim 20 M_{J}\right)$ such that, for a planet with lower mass the eccentricity would be damped, otherwise the eccentricity is excited to values of $0.1 \sim 0.25$. They suggested that the transition mass might be reduced into the range of the observed extrasolar planets at a very low disc viscosity.

\subsection{Interactions between planets and planetesimal disk}

Migration due to interactions between planets and planetesimal disk is well studied in the evolution of outer solar system (e.g., Malhotra 1993, Hahn \& Malhotra, 1999). The direction of the planet migrations is mainly determined by the averaged angular momentum $H=\sqrt{a\left(1-e^{2}\right)}$ of the planetesimals. For planetesimals with $H$ greater than that of the planet's $H_{p}$, they have a larger average tangential velocity relative to the orbital motion of the planet, thus the result of close encounters between the planetesimals and the planet is to increase the angular momentum of the planet, which leads to an outward migration of the planet (Ida et al. 2000, Gomes, Morbidelli, Levison 2004). Since the encounters between planetesimals and planets are more or less erratic, the migration caused by interactions between planets and planetsimal disk is not smooth(Ida et al. 2000, Zhou et al. 2002).

Murray et al. (1998) proposed that resonant interactions between the planet and the planetesimals can remove angular momentum from the planetesimals, increasing their eccentricities. Subsequently, the planetesimals either collide with or are ejected by the planet, reducing the semi-major axis of the planets. If the surface density for the planetesimals is above some critical values, an instability would occur so that the planet can migrate inward with by a large amount. However, the critical surface density for the planetesimals should be $\sim 200 \mathrm{gcm}^{-3} \mathrm{r}^{-3 / 2}$, which is an order of magnitude larger than that of the minimum solar nebula.

\section{Dynamics of Multiple Planet Systems}

The evolution of a multiple planet system under their mutual attractions is a paradigm of the classical $\mathrm{N}$ body problem, which is not integrable for $N \geqslant 3$. The study of the stability of planet systems can give constrains on the parameters of the planets, especially the mass of planets, which are determined up to a $\sin i$ factor from the Doppler survey. For the convenience of stability study, multiple planet systems can be classified in three types (e.g., Barnes \& Quinn 2004): resonant, interacting and hierarchical systems. Resonant systems contain planets in lower order mean motion resonances (MMR). Interacting system contain planets that are not in lower order mean motion resonances but are separated with period ratio less than $10: 1$. Hierarchical systems contain planets with 
Table 1. Parameters of the planets in Resonances

\begin{tabular}{|c|c|c|c|c|c|c|}
\hline star & $\begin{array}{l}M_{*} \\
\left(M_{\odot}\right)\end{array}$ & $\begin{array}{l}M_{p} \sin i \\
\left(M_{\text {Jup }}\right)\end{array}$ & $\begin{array}{l}a \\
(\mathrm{AU})\end{array}$ & $\begin{array}{l}\text { Period } \\
\text { (days) }\end{array}$ & eccentricity & comment \\
\hline $55 \mathrm{Cnc} \mathrm{b}$ & 1.03 & 0.84 & 0.115 & 14.65 & 0.02 & $3: 1 \mathrm{MMR}+\mathrm{AC}$ \\
\hline $55 \mathrm{Cnc} \mathrm{c}$ & 1.03 & 0.21 & 0.24 & 44.28 & 0.34 & $3: 1 \mathrm{MMR}+\mathrm{AC}$ \\
\hline GJ876 c & 0.32 & 0.56 & 0.13 & 30.1 & 0.27 & $2: 1 \mathrm{MMR}+\mathrm{AC}$ \\
\hline GJ876 b & 0.32 & 1.89 & 0.21 & 61.0 & 0.10 & $2: 1 \mathrm{MMR}+\mathrm{AC}$ \\
\hline HD82943 c & 1.05 & 1.85 & 0.75 & 219.4 & 0.54 & $2: 1 \mathrm{MMR}+\mathrm{AC}$ \\
\hline HD82943 b & 1.05 & 1.84 & 1.18 & 435.1 & 0.41 & $2: 1 \mathrm{MMR}+\mathrm{AC}$ \\
\hline $47 \mathrm{UMa} b$ & 1.03 & 2.54 & 2.09 & 1089 & 0.06 & ASR \\
\hline $47 \mathrm{UMa} \mathrm{c}$ & 1.03 & 0.76 & 3.73 & 2594 & 0.1 & ASR \\
\hline Ups And c & 1.30 & 1.89 & 0.829 & 241.5 & 0.28 & ASR \\
\hline Ups And d & 1.30 & 3.75 & 2.53 & 1284 & 0.27 & ASR \\
\hline HD12661 b & 1.07 & 2.3 & 0.82 & 263.6 & 0.33 & ASR \\
\hline HD12661 c & 1.07 & 1.5 & 2.46 & 1530 & 0.2 & ASR \\
\hline
\end{tabular}

Note : MMR-mean motion resonance; AC-apsidal corotation; ASR-apsidal secular resonance. Data of HD82943 from Mayor et al. 2004, others from http://exoplanets.org/

a period ratio grater than $10: 1$, and can be approximated as dynamically decoupled systems, provided that their eccentricities are not very large.

According to the above definition, our solar system is an interacting system. For the 11 confirmed multiple extra-solar planetary systems to date, 3 pairs of planets are confirmed in mean motion resonances (Fischer et al. 2003): HD82943, GJ876, 55 Cancri b and c, see Table 1. The HD160691 system, with uncertain parameters, could be most probably in a 2:1 resonance (Bois et al. 2003). Interacting systems include 3 systems: Ups And (c-d), HD12661, 47 Uma. 5 systems are hierarchical: HD38529, HD74156, HD168443, HD37124, HD169830. The last two systems are marginal hierarchical, with period ratios 9.8 and 9.3, respectively. As the eccentricities of planet orbits in these two systems are large, their stability need inspection. The outmost planet of 55 Cancri and the innermost planet of Ups And are hierarchical with other planets in the same system.

\subsection{Resonant Systems}

Mean motion resonance is an important mechanism that leads to stable configurations for nearby planets. Among the three confirmed pairs of planets in resonances, 5 planets are in orbits with large eccentricities, which is in contradiction with our knowledge about stability. Thus a natural question is, can stable periodic solutions exist with high eccentricities, corresponding to equilibriums of mean motion resonances? Hadjidemetriou (2002) gives a positive answer. Let us take the $2: 1$ resonance as an example. Define the corresponding two resonance angles as

$$
\begin{aligned}
& \theta_{1}=2 \lambda_{2}-\lambda_{1}-\varpi_{1}, \\
& \theta_{2}=2 \lambda_{2}-\lambda_{1}-\varpi_{2}, \\
& \sigma=\theta_{2}-\theta_{1}=\varpi_{1}-\varpi_{2}=\Delta \varpi .
\end{aligned}
$$

Resonant solutions with $\theta_{1}=0, \sigma=0$ are symmetrical periodic solutions with apsidal corotation $(\mathrm{AC})$ in a coordinate system comoving with one planet. Using the continuation method of the periodic orbits, Hadjidemetriou (2002), Hadjidemetriou \& Psychoyos (2003) studied the families of periodic orbits near the 2:1 and 3:1 resonances in a 2-planet systems with applications to the GJ876 and HD82943 systems. Stable resonance orbits are found at large eccentricities with $e_{1}>e_{2}$, in accordance with the two systems. 
The existence of asymmetric libration solutions $\left(\theta_{1} \neq 0, \pi, \sigma \neq 0, \pi\right)$ has been shown by Beaugé, Ferraz-Mello, and Michtchenko (2003), with a new perturbation method developed by Beaugé and Michtchenko (2003). The method allows us to study analytically the evolution of extrasolar planets on high eccentricity orbits. They find that the existence of stable periodic orbits depends on the mass ratio of the two planets $m_{1} / m_{2}$, thus it gives a constraint for the mass of the observed planets. They also find that the evolutionary track of GJ876 and HD82943's eccentricity coincide with the lines of stable 2:1 resonance solutions for the observed mass ratio. This gives a strong evidence of a migration history for these systems. This method is applied to the 55 Cancri system: the two inner planets are in 3:1 resonance and also in apsidal corotation (Zhou et al. 2004). From hydrodynamical simulations, tidal migrations of planets pairs in 55 Cancri can lead to a trapping of both 3:1 resonance and apsidal corotation (Kley 2002).

One of the remaining problems concerning the migration evolution of the resonant systems is the damping of their eccentricities. Suppose the planets are initially in circular orbits, when they migrate under the tidal interaction of either gaseous disk or planetesimal disk, their eccentricities will increase inevitably (e.g., Lee and Peal 2002). So a damping mechanism for the eccentricity are required to fit the observed values. Lee and Peal (2002) find that a damping rate with

$$
\frac{\dot{e}_{i}}{e_{i}}=-K\left|\frac{\dot{a}_{i}}{a}\right|, \quad(i=1,2)
$$

and $K \approx 100$ is required to account for the observed eccentricities of the two planets in GJ876 systems. Though this type of damping rate is coincident in form with that from disk-planet interactions, the required value of $K$ is much larger than that obtained from the density wave theory.

\subsection{Interacting systems}

For the interacting systems, apsidal secular resonance (ASR) could be one of the dynamical effects that enforce the stability of planetary systems. An ASR occurs when the secular motion of two planets' longitudes of the perihelion $\left(\varpi_{1}\right.$ and $\left.\varpi_{2}\right)$ have almost the same frequency, thus $d(\Delta \varpi) / d t \approx 0$. Planets in aligned ASR can reduce the possibility of close encounters, thus ASR can stabilize the interacting planets. An impulsive perturbation (e.g. by ejection of other planets) which increases the eccentricity of one planet can possibly lead two planets into ASR (Malhotra 2002). All the 3 pairs of planets in the present observed interacting systems are in ASR (see Table 1): Ups And c-d, 47UMa, HD12661. According to the linear secular perturbation theory, whether two planets in ASR depends only on $m_{1} / m_{2}, a_{1} / a_{2}, e_{1} / e_{2}$ and $\Delta \varpi$ (Zhou and Sun 2003).

The HD12661 system is the first extra-solar planetary system found to have two planets in anti-aligned $\operatorname{ASR}(\Delta \varpi=\pi)$. Lee and Peale (2003) use octopole-level secular perturbation theory to study the secular evolution of a planetary system. They find that, when the angular momentum ratio $L_{1} / L_{2} \approx\left(m_{1} / m_{2}\right)\left(a_{1} / a_{2}\right)^{1 / 2}$ is equal to some critical values, the phase space of the secular system is occupied by apsidal librations. They find HD12661 system is close to this critical configurations, thus the secular system of HD12661 is stable. However, as a general three-body system, the present observed HD12661 system is on the border between chaos and regular motion (Kiseleva-Eggleton et al. 2002, Zhou \& Sun 2003).

\subsection{Stable region}

Most works are devoted to the stability study of the observed multiple planet systems from the fitted initial conditions. The general N-body model is used during the numerical 
integrations, with the auxiliary computation of Lyapunov characteristic numbers (LCN), Fast Lyapunov Indicator (FLI) developed by Froeschlé, Lega \& Gonczi (1997) and the MEGNO technique by Cincotta \& Simó (2000). Barnes \& Quinn find that the stable regions of the two resonant systems, HD82943 (with old data from 'Extrasolar Planets Encyclopaedia') and GJ876, are very narrow (Barnes \& Quinn 2004). This indicates that the HD82943 and GJ876 systems are protected only by the mean motion resonances, and most likely the present large eccentricities are the consequence of planet migrations. For the unconfirmed HD160691 system, the planets can be stable only in a 2:1 MNR (Bois et al. 2003), which shows HD160691 system should be a member of the resonant systems as well.

For the interacting systems, two systems (Ups And , 47 Uma) have border stable regions (Barnes \& Quinn 2004), thus they could be stable even without the apsidal secular resonances, and the observed eccentricity could be the results of planet scattering. The HD12661 system is on the border of chaotic and regular motions as mentioned before (Zhou \& Sun 2003,Barnes \& Quinn 2004).

The stability of the marginal hierarchical system HD169830 is studied by Goździewski \& Konacki (2004), which shows the present system is in a wide stable zone, thought the planets have large eccentricities. For another marginal hierarchical system HD37124, Kiseleva-Eggleton et al. (2002) found the present configuration is near the border of a wide stable region. Dvorak et al. (2003) find there is difficult to host planets in the habitable zone of the hierarchical system HD74156. A systematical study of dynamics of binary stars planetary system have been done by their group (e.g., Pilat-Lohinger, Funk, Dvorak, 2003).

\subsection{Onset of instability}

Since generally a Hamiltonian system with more than two degrees of freedom is not integrable, instability will arise for a planet system with two planets or more. Fortunately, for a planet system with sufficient small planet mass and sufficient large separations, the speed of orbital diffusion in the action space (corresponds to angular momentum and energy, etc.) could be very small. Knowing the time scale over which instability may occur would be helpful to understand the time scale of the excitation of the orbital eccentricities by scattering among planets.

For a planet system with two planets initially on circular orbits, the system will be Hill stable (stable against close approaches for all time) if the relative orbital separation of the two planets $\left(\delta=\left(a_{2}-a_{1}\right) / a_{1}\right)$ fulfils, $\delta>2.4\left(\mu_{1}+\mu_{2}\right)^{1 / 3}$, where $\mu_{i}=$ $m_{i} / m_{*}(\mathrm{i}=1,2)$ (Gladman, 1993). However, when the planet number is larger than 2 , things become quite different. Suppose $N$ planets with equal mass $m$ initially on circular orbits, and the separations are equal when scaled with their mutual Hill's radius, $R_{H}=(2 \mu / 3)^{1 / 3}\left(a_{i}+a_{i+1}\right) / 2,(i=1, \ldots n-1)$. Chambers, Wetherill and Boss (1996) found the crossing time $T_{c}$, defined as the minimum time that orbital crossing is expected, could be approximated by $\log \left(T_{c}\right)=b\left(\Delta a_{0} / R_{H}\right)+c$, where $b(>0), c$ are constants, $\Delta a_{0}=a_{i+1}-a_{i}$, and $T_{c}$ is in unit of years. Thus whenever the planet number is larger than 2, there is no Hill-stable region in the phase space so that $T_{c} \rightarrow \infty$. An important result is that $b, c$ seem to have little dependence on $N$ when $N \geqslant 5$. Yoshinaga, Kokubo \& Makino (1999) extended the study to the case when planets are initially in elliptic and inclined orbits. An extension to a much wider initial separations shows that, the orbital crossing time of planets in initially circular orbits can be expressed as (Zhou, Lin, Sun, in preparation):

$$
\log \left(T_{c}\right) \approx-2-\frac{2}{7} \log \mu+(19+1.2 \log \mu) \log \left(\frac{\Delta a_{0}}{2.3 R_{H}}\right), \quad\left(\Delta a_{0}>2.3 R_{H}\right)
$$


where $T_{c}$ is in the unit of years. The crossing time for planet initially in circular and non circular orbits are shown in Fig.2 (the initial eccentricity $e_{0}$ is scaled by $h=\left(a_{i+1}-\right.$ $\left.\left.a_{i}\right) /\left(a_{i+1}+a_{i}\right)\right)$. According to (4.3) and Fig.2, there exist systems that are dynamically unstable but the time scale over which such instability occurs is much longer than the physical age of the system, thus it is "effectively stable".

On the other hand, for planets with small separations, the orbital crossing time can be very short. According to (4.3), the instability time scale will $\sim 10^{6.7}$ years for $N(N>3)$ protoplanets with mass $\mu=10^{-7}$ and $\Delta a_{0} / R_{H}=10$. Thus the excitation of planet eccentricities can occur within a short time scale. But as the protoplanets have larger masses, the excitation of the eccentricities require a longer time.
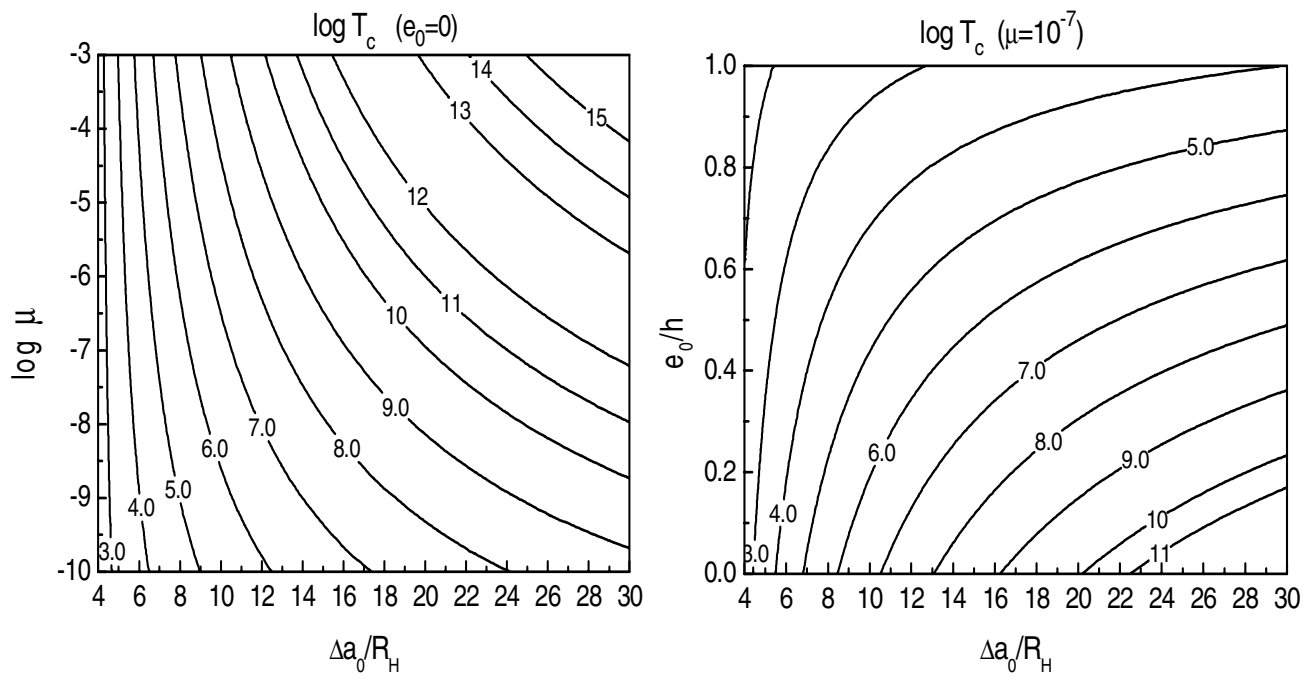

Figure 2. The orbital crossing time of multiple planet systems with equal planet masses and scaled separations (see text). (a) planets are initially in circular orbits. (b) Planets with $\mu=10^{-7}$.

\section{Summary}

The dynamical evolution of the extra-solar planetary systems is a complicate process involving planet-disk interactions and interplanetary scattering. According to the present knowledge, we have the following conclusions:

(1) For the observed extra-solar planets with short period orbits, migration is believed to occur after the planets are formed. The type I migration rate given by the linear density wave theory would be too fast. The lack of large planets with short period orbits may be due to either the inefficient mass accretion for planets at small period orbits, or due to the inefficient (or too efficient) migration of large planets. If the latter scenario is right, we need a mechanism to generate a mass-dependent migration.

(2) For single planet systems with massive planet mass (of the order of Jupiter mass), the interactions with gaseous disk can excite the eccentricities of the planet orbits, However, the required minimum mass of planet is too large as found by some numerical simulations. Other dynamical processes, e.g., scattering with smaller, unseen planets, can also generate the eccentricity.

(3) For multiple planet systems, the excitation of eccentricity can either by interplanetary scattering, which may lead to an erratic change of the eccentricity, or by 
adiabatic migration due to interactions with remnant gaseous disk, which leads to a gradual increase of eccentricity. The eccentricities of planets in interacting systems and hierarchical systems can be excited by the former mechanism. On the other hand, the eccentricities of planets in resonant systems are most probably excited by the latter mechanism during the migration, being trapped into resonance. However, in the resonance trapping case, we still need a mechanism to keep the eccentricities as the observed values after they are excited.

Our current knowledge is limited due to the insufficient observations, and it's still too early to say that our solar system is very special among the known planet systems. With the development of the observation technics we can get more knowledge of the possible configurations of planetary systems, and celestial mechanics will become more and more important in the study of dynamics of the extrasolar planetary systems.

\section{Acknowledgements}

This work is supported by the Natural Science Foundation of China under Grant No.19903001 and the Special Funds for Major State Basic Research Project(G200077303).

\section{References}

Artymowicz, P., 1993, Astrophys. J., 419, 155

Barnes, R. \& Quinn, T., 2004, Astrophys. J., 611, 494

Beaugé, C., Ferraz-Mello, S., \& Michtchenko, T.A., 2003, Astrophys. J., 593, 1124

Beaugé, C. \& Michtchenko, T.A., 2003, Mon. Not. R. Astron. Soc., 341, 760

Bois, E., Kiseleva-Eggleton, L., Rambaux, N., \& Pilat-Lohinger, E., 2003, Astrophys. J., 598, 1312

Bouchy, F., Pont, F., Santos, N.C., Melo, C., Mayor, M., Queloz, D., \& Udry, S., 2004 Astron. Astrophys. 421, L13

Bryden,G., Chen, X., Lin, D.N.C., Nelson, R.P., \& Papalozou, J.C.B., 1999 Astrophys. J., 514, 344

Chambers, J.E., Wetherill, G.W., \& Boss, A.P., 1996, Icarus, 119, 261

Cincotta, P.M. \& Simó, C., 2000, A\&AS, 147, 205

Dobbs-Dixon, I, Lin, D.N.C. \& Mardling, R. A., 2004, Astrophys. J., 610, 464

Dvorak, R., Pilat-Lohinger, E., Funk, B., \& Freistetter, F., 2003 Astron. Astrophys., 410, L13

Froeschle, C., Lega, E., \& Gonczi, R., 1997, Celest. Mech. Dyn. Astron. 67, 41

Fischer, D.A., Marcy, G.W., Butler, R.P., Vogt, S.S., Henry, G.W., Pourbaix, D., Walp, B., Misch, A.A., \& Wright, J.T., 2003, Astrophys. J., 586, 1394

Gladman, B., 1988, Icarus, 106, 247

Goldreich, P. \& Sari, R.,2003, Astrophys. J., 585, 1024

Goldreich, P. \& Tremaine, S., 1979, Astrophys. J., 233, 857

Goldreich, P. \& Tremaine, S., 1980, Astrophys. J., 241, 425

Gomes, R.S., Morbidelli, A., \& Levison, H.F., 2004, Icarus, 170, 492

Goździewski, K. \& Konacki, M., 2004, Astrophys. J., 610, 1093

Hadjidemetriou, J.D. \& Psychoyos, D., 2003, in: G. Contopoulos, N. Vogglis (eds.), Galaxies and Chaos, Lecture Notes in Physics, Vol. 626, p. 412

Hadjidemetriou, J.D., 2002, Cel. Mech. Dyn. Astron., 83, 141

Hahn, J.M. \& Malhotra, R., 1999, Astron. J., 117, 3041

Ida, S., Bryden, G., Lin, D.N.C., \& Tanaka, H., 2004, Astrophys. J., 534, 428

Ivanov, P.B., Papaloizou, J.C.B., \& Polnarev, A.G., 1999, Mon. Not. R. Astron. Soc., 307, 79

Kley, W., 1999, Mon. Not. R. Astron. Soc. 303, 696

Kley, W., 2003, Cel. Mech. Dyn. Astron. 87, 85

Koller, Josef; Li, Hui; Lin, \& Douglas, N.C., 2003, Astrophys. J., 596, L01

Kokubo, E.K. \& Ida, S., 1998, Icarus, 131, 171 
Konacki, M., Torres, G., Sasselov, D.D., Pietrzynski, G., Udalski, A., Jha, S., \& Ruiz, M.T., Gieren, W., \& Minniti, D., 2004, Astrophys. J., 609, L37

Kuchner, M.J. \& Lecar, M., 2002, Astrophys. J., 574, L87

Kiseleva-Eggleton, L., Bois, R., Rambaux, N., \& Dvorak, R., 2002, Astrophys. J., 578, L145

Laughlin, G., Steinacker, A., \& Adams, R., 2004, Astrophys. J., 608, 489

Lee, M.H. \& Peale, S.J. 2002, Astrophys. J., 567, 596

Lee, M.H. \& Peale, S.J. 2003, Astrophys. J., 592, 1201

Lin, D.N.C, Bodenheimer, P., \& Richardson, D.C., 1996, Nature, 380, 606

Lin, D.N.C. \& Papaloizou, J., 1979, Mon. Not. R. Astron. Soc., 186, 799

Lin, D.N.C. \& Papaloizou, J.C.B., 1993, in: E.H. Levy, \& J.I. Lunine (eds.), Protostars and Planets III, Univ. Arizona Press, p. 749

Lin, D.N.C., Papaloizou, J.C.B., Terquem, C., \& Bryden, G., 2000 in: V. Mannings, A.P. Boss, \& S.S. Russell (eds.), Protostars and Planets IV, Univ. Arizona Press, p. 1111

Malhotra, R., 1993, Nature, 365, 819

Malhotra, R., 2002, Astrophys. J., 575, L33

Marcy, G.W., Bulter, R.P., Fischer, D.A. et al. 2003, in: D. Deming, \& S. Seager (eds.), Scientific Frontiers in Research on Extrasolar Planets, ASP Conferences Series, Vol. 294, p. 1

Mayor, M. \& Queloz, D., 1995, Nature 378, 355

Mayor, M., Udry, S., Naef, D., Pepe, F., Queloz, D., Santos, N.C., \& Burnet, M., 2004, Astron. Astrophys., 415, 391

Matsuyama, I., Johnstone, D., \& Murray, N., 2003, Astrophys. J., L143

Murray, N., Hansen, B., Holman, M., \& Tremaine, S., 1998, Science, 279, 69

Nagasawa, M., Lin, D.N.C., \& Ida, S., 2003, Astrophys. J., 586, 1374

Nelson, R.P. \& Papaloizou, J.C.B., 2004, Mon. Not. R. Astron. Soc., 350, 849

Nelson, R.P., Papaloizou, J.C.B., Masset, F., \& Kley, W., 2000, Mon. Not. R. Astron. Soc., 318, 18

Papaloizou, J.C.B., Nelson, R.P., \& Masset, F., 2001, Astron. Astrophys., 366, 263

Pilat-Lohinger, E., Funk, B., \& Dvorak, R. 2003, Astron. Astrophys., 400, 1085

Rafikov, R.R., 2002, Astrophys. J., 572, 566

Shakura, N.I. \& Sunyaev, R.A., 1973, Astron. Astrophys., 24, 337

Terquem, C., Papaloizou, J.C.B., Nelson, R.P., \& Lin , D.N.C., 1998, Astrophys. J., 502, 788

Torres, G., Konacki, M., Saaaelov, D.D., \& Jha, S., 2004, Astrophys. J., 609, 1071

Udry, S., Mayor, M., \& Queloz, D. 2003, in: D. Deming, \& S. Seager (eds.), Scientific Frontiers in Research on Extrasolar Planets, ASP Conferences Series, Vol. 294, p. 17

Veras, D. \& Armitage, P.J., 2004, Mon. Not. R. Astron. Soc., 347, 613

Ward, W.R., 1988, Icarus, 73,330

Ward, W.R., 1997a, Icarus, 126,261

Ward, W.R., 1997b, Astrophys. J., 482, L211

Ward, W.R. \& Hahn, J.M., 2000, in: V. Mannings, A.P. Boss, \& S.S. Russell (eds.), Protostars and Planets IV, Univ. Arizona Press, p. 1111

Wolszcan, A. \& Frail, D.A., 1992, Nature 355, 145

Yoshinaga, K., Kokubo, E., \& Makino, J., 1999, Icarus, 139, 328

Zhou, J.L. \& Sun, Y.S., 2003, Astrophys. J., 598, 1290

Zhou, L.Y., Sun, Y.S., Zhou, J.L., Zheng, J.Q., \& Valtonen, M., 2002, Mon. Not. R. Astron. Soc., 336,520

Zhou, L.Y., Lehto, H.J., Sun, Y.S., \& Zheng, J.Q., 2004, Mon. Not. R. Astron. Soc., 350, 1495 